\title{
Dissociative recombination exalts molecular growth in $\mathrm{N}_{2} / \mathrm{CH}_{4}$ plasmas
}

\author{
P. Pernot ${ }^{1, \mathrm{a}}$, Z. Peng ${ }^{1}$, S. Plessis ${ }^{1}$ and N. Carrasco ${ }^{2,3}$ \\ ${ }^{1}$ Laboratoire de Chimie Physique, UMR 8000, CNRS, Univ Paris-Sud, 91405 Orsay, France \\ ${ }^{2}$ Université de Versailles Saint-Quentin, CNRS/INSU, LATMOS, UMR 8190, 78280 Guyancourt, \\ France \\ ${ }^{3}$ Institut Universitaire de France, 103 Bvd. St-Michel, 75005 Paris, France
}

\begin{abstract}
We present a comparison of the outcomes of two models of $\mathrm{N}_{2} / \mathrm{CH}_{4}$ VUV plasmas in two sets of conditions representative of a laboratory experiment and of Titan's ionosphere. The "FullDR" model incorporates all available experimental data on dissociative recombination od $\mathrm{C}_{x} \mathrm{H}_{y} \mathrm{~N}_{z}^{+}$ions thanks to the probabilistic tree technique recently developed in our group, whereas the "Hloss" model implements the H-loss pathways used by default in many models. We show that the FullDR model enhances globally the production of neutrals and ions, through the production of $\mathrm{C}_{2} \mathrm{H}_{x}$ fragments. These species are efficient promoters of molecular growth, notably in the higher pressure conditions of the laboratory experiment.
\end{abstract}

\section{Introduction}

Complete sets of dissociative recombination branching ratios (BR) are known for only a handful of ions $[1,2]$. In most cases, notably for $\mathrm{C}_{x} \mathrm{H}_{y} \mathrm{~N}_{z}^{+}$ions, the distribution of $\mathrm{H}$ atoms amongst the fragments is not experimentally reachable. Without this information, the chemical identity of the fragments is unknown, and therefore their further reactivity. This situation has for long prevented the use of a large corpus of experimental BR data in detailed chemistry models, and many modelers have instead implemented a default "H-loss" scheme, where the parent ions looses a single H atom [3-8]. The "H-loss" scheme is not supported by the experiments: many ions present major dissociation pathways involving heavy atoms bond breaking, for instance $\mathrm{C}_{4} \mathrm{H}_{8}^{+}$[9] or $\mathrm{HC}_{3} \mathrm{~N}^{+}$and other nitrile ions [10].

We have solved this problem by proposing a probabilistic representation enabling to handle incomplete BR sets $[11,12]$. For the first time, we have been able to take advantage of all available BR data for $\mathrm{C}_{x} \mathrm{H}_{y} \mathrm{~N}_{z}^{+}$ions in a model of the $\mathrm{N}_{2} / \mathrm{CH}_{4}$ plasma in Titan's ionosphere [13]. This preliminary model considered a fixed neutral bath composition, and did not enable us to quantify direclly the impact of these new data on the predicted mole fractions of neutral species.

Recently, a new fully coupled ion-neutrals model has been developed and used to study the impact of different physical and chemical factors on the molecular growth in $\mathrm{N}_{2} / \mathrm{CH}_{4}$ photochemical plasmas

\footnotetext{
${ }^{a}$ Corresponding author: pascal . pernot@u-psud.fr
}

This is an Open Access article distributed under the terms of the Creative Commons Attribution License 4.0, which permits unrestricted use, distribution, and reproduction in any medium, provided the original work is properly cited. 
Table 1. Physical conditions of the two scenarii discussed in the text. $P$ is the pressure in the reactor, $T$ is the gas temperature and $I_{0}$ is the integrated photon flux between 50 and $200 \mathrm{~nm}$. DISCO is a VUV beamline at the French SOLEIL synchrotron [20].

\begin{tabular}{lcc}
\hline & APSIS & "Titan" \\
\hline$P / \mathrm{Pa}$ & $7 \times 10^{2}$ & $7 \times 10^{-4}$ \\
$T / \mathrm{K}$ & 300 & 150 \\
Spectrum & DISCO & Solar \\
$I_{0} / \mathrm{ph}_{\mathrm{m}} \mathrm{m}^{-2} \cdot \mathrm{s}^{-1}$ & $2 \times 10^{12}$ & $1 \times 10^{9}$ \\
\hline
\end{tabular}

$[14,15]$, with a specific focus on synchrotron-based experimental setups designed to mimic Titan ionospheric chemistry [17, 18], and notably the APSIS reactor [19]. Of particular interest is the role of ion chemistry on the formation of heavy N-bearing molecules in ionospheric conditions [16].

The present article uses this model to show how dissociative recombination impacts molecular growth in these plasmas and to compare the predictions of our "FullDR" model with those of the corresponding "H-loss" model. The data needs resulting from this study are also underlined.

\section{The model}

A photochemical-transport model has been designed to represent different approximations of the APSIS reactor [19], from OD (1-cell model) to $2 \mathrm{D}$ (cylindrical symmetry). In order to be able to manage the computational cost of the probabilistic tree representation of dissociative recombination BR sets [11], we use here a 1-cell geometry. The model assumes no transport and a uniform spatial distribution of all species, but takes into account the gas inflow (injection) and outflow (pumping) and the radiative transfer. Details of the model are presented elsewhere $[14,15]$.

Simulations are performed in two sets of conditions representative of (1) the APSIS experiment; and (2) a crude proxy of Titan's ionosphere (noted "Titan" thereafter). In both cases a 90:10 $\mathrm{N}_{2} / \mathrm{CH}_{4}$ mixture is considered. The two scenarii differ in several parameters, as reported in Table. 1: (i) the synchrotron DISCO flux at SOLEIL synchrotron has a rather flat wavelength distribution, in contrast with the solar spectrum mainly peaked at Lyman- $\alpha$ [19]; (ii) APSIS experiments are led at room temperature, whereas a reference temperature of $150 \mathrm{~K}$ is used to represent Titan's ionospheric conditions; and (iii) a large $\left(10^{6}\right)$ ratio between the APSIS and "Titan" pressure conditions, which is the major source of difference in the products distributions and abundances $[14,15]$.

The chemical scheme incorporates the complete neutral chemistry by Hébrard et al. [21, 22], with adapted photodissociation branching ratios following Gans et al. [23]. The model implements the ion chemistry database established by Carrasco et al. [24] and Plessis et al. [11], involving C, H and N. Oxygen chemistry has been excluded because it does not play a significant role in APSIS photochemistry [19].

The "FullDR" model considers 244 species (125 neutrals and 119 ions) reacting through 1708 reactions (33 photolysis, 415 bimolecular reactions, 82 termolecular reactions, 574 dissociative recombinations, 604 ion-molecule reactions). The rate parameters of these processes are all uncertain (some of them unknown). They are represented by random variables with prescribed distributions, following Hébrard et al. [21, 22], Carrasco et al. [24] and Plessis et al. [11].

A derived "Hloss" model implements the "H-loss" scheme for dissociative recombination, where appropriate. In this scenario, the 448 dissociative recombination reactions coded as probabilistic trees for 58 ions are reduced to 63 reactions $[14,15]$. 


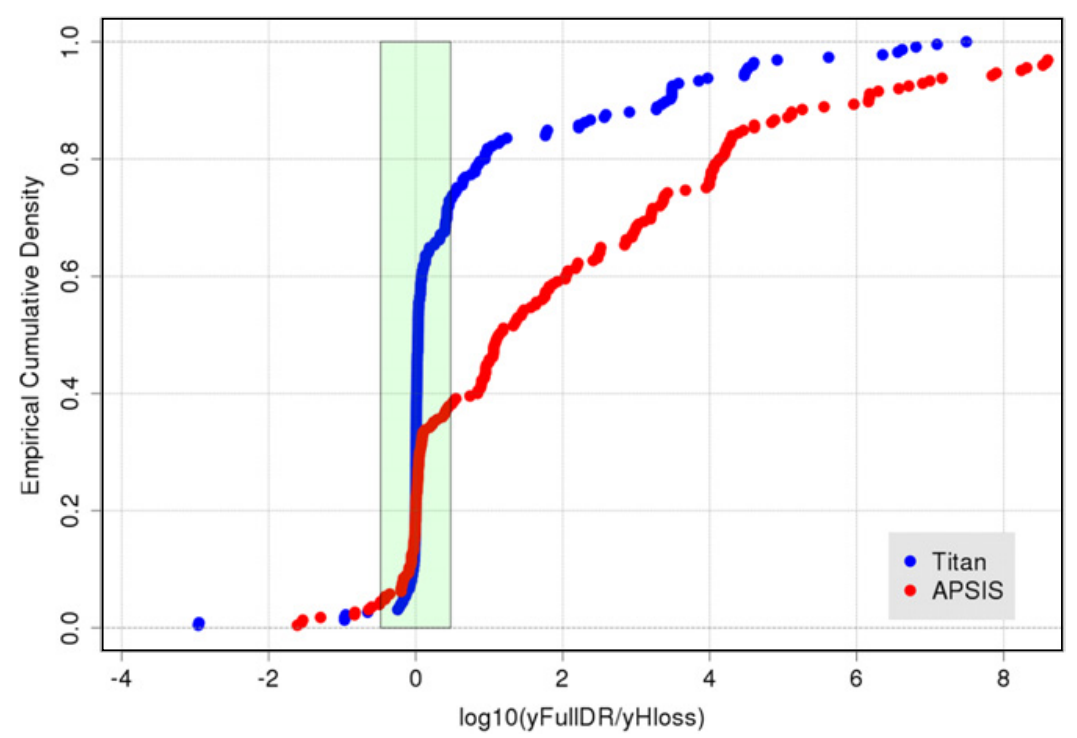

Figure 1. Impact of the dissociative recombination branching ratios representation on the mole fractions of the products of a $\mathrm{N}_{2} / \mathrm{CH}_{4}$ photochemical plasma in two physical setups (see text). The shaded zone depicts the $95 \%$ confidence interval on the plotted values: the points outside of this zone can be considered as significantly different from zero.

\section{Results and discussion}

A sample of 500 realizations of the uncertain dissociative recombination rate parameters and branching ratios has been generated. All other parameters have been kept fixed. The photochemical code has been run for each realization, and the corresponding sample of stationary state mole fractions has been used to build the Figs. 1-3 discussed below.

To assess the global impact of the improved dissociative recombination description in the "FullDR" model when compared to the "Hloss" model, we evaluated for each species the ratio of the sample average mole fractions $\left(y_{F u l l D R} / y_{\text {Hloss }}\right)$. The empirical cumulative density function built from the values for all the species is presented in Fig. 1. In the APSIS setup at least $60 \%$ of the species have exalted mole fractions, whereas this ratio is about $30 \%$ in the "Titan" conditions. In both cases, the enhancement factors can reach very large values: more than $10^{2}$ for $40 \%$ of the species in the APSIS conditions (15\% in "Titan"). The prediction by Plessis et al. [13] of a global increased production mediated by the reactive fragments of dissociative recombination is therefore verified.

The mole fractions probability densities for selected species have been plotted for both BR models in the APSIS conditions in Fig. 2. The data for the "FullDR" case are systematically and significantly larger than for the "Hloss" scenario. A few species $\left(\mathrm{C}_{2} \mathrm{H}_{2}, \mathrm{NH}_{3}, \mathrm{C}_{6} \mathrm{H}_{6}\right.$ and $\left.\mathrm{HC}_{3} \mathrm{~N}\right)$ considered as emblematic in Titan's ionosphere are surrounded by red lines. The impact of dissociative recombination $\mathrm{BR}$ representation on most species is very strong (more than one order of magnitude). Flux analysis shows that the formation of $\mathrm{C}_{2} \mathrm{H}_{x}$ unsaturated fragments through dissociative recombination of $\mathrm{C}_{3} \mathrm{H}_{y}^{+}$ ions is a major contribution to this production exaltation. In this model, $30 \%$ of $\mathrm{C}_{2} \mathrm{H}_{2}$ comes from $\mathrm{C}_{3} \mathrm{H}_{7}^{+}$, and $\mathrm{C}_{2} \mathrm{H}_{2}$ is known to contribute very efficiently to molecular growth $[14,15,26]$. These pathways are absent from the "Hloss" model, although a full set of branching ratios has been measured [25]. This effect of an increased $\mathrm{C}_{2} \mathrm{H}_{2}$ production is directly visible on the mole fractions of $\mathrm{C}_{6} \mathrm{H}_{6}$ and $\mathrm{HC}_{3} \mathrm{~N}$.

One observes also larger uncertainty for the "FullDR" data, which is due to the assimilation of unknown BR sets in this model. The very large uncertainty of most N-bearing species can be traced 


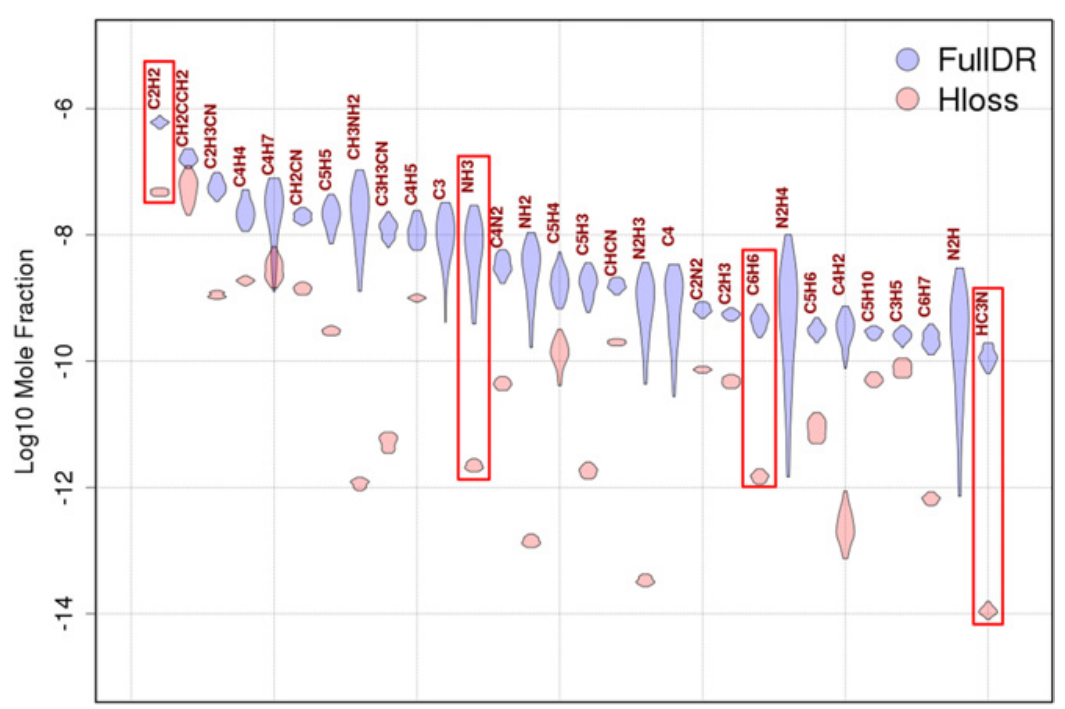

Figure 2. Probability density plots for the stationary mole fractions of selected species in the APSIS physical conditions (see Table 1).

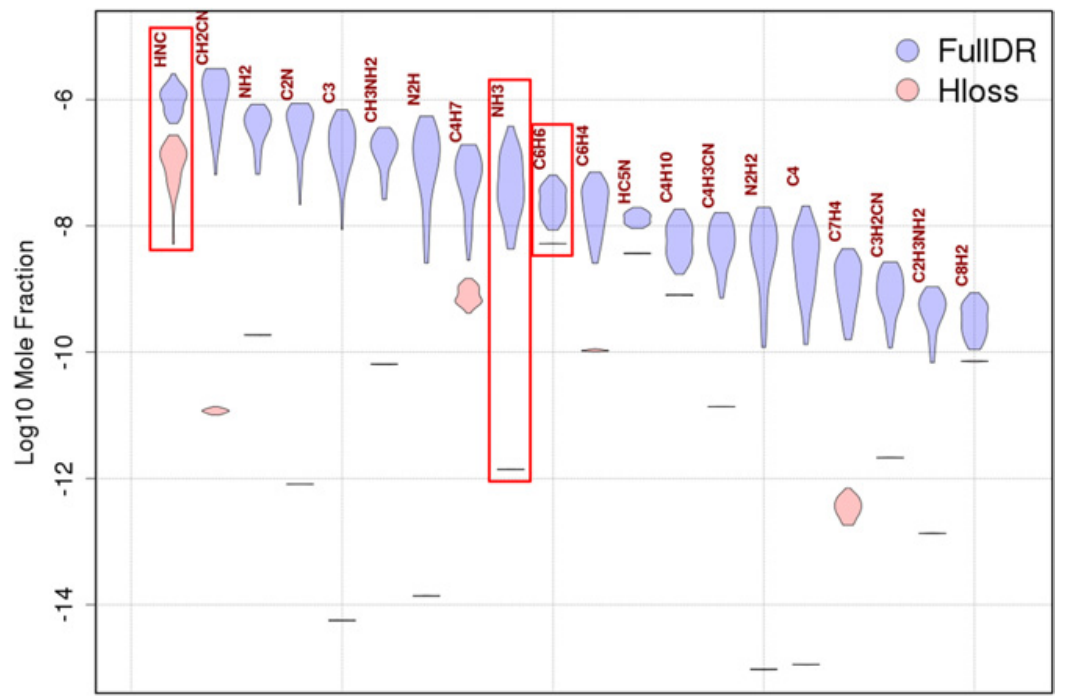

Figure 3. Probability density plots for the stationary mole fractions of selected species in the "Titan" physical conditions (see Table 1).

back to the dissociative recombination of $\mathrm{CH}_{2} \mathrm{NH}_{2}^{+}$, for which rate constant and branching ratios are missing $[11,27]$. The enhanced $\mathrm{NH}_{3}$ mole fraction is also linked to this process, through the $\mathrm{NH}_{2}$ fragment.

A similar analysis has been done for "Titan" conditions in Fig. 3. The impact of the model is very strong for $\mathrm{NH}_{3}$, and remains significant for $\mathrm{C}_{6} \mathrm{H}_{6}$, but there is no visible impact on $\mathrm{C}_{2} \mathrm{H}_{2}$ (not shown). In this setup, the contribution of dissociative recombination to the formation of $\mathrm{C}_{2} \mathrm{H}_{2}$ and other unsaturated $\mathrm{C}_{2} \mathrm{H}_{x}$ species is negligible. Conversely, the role of dissociative recombination is dominant for many of 
the hydrocarbons (e.g. $\mathrm{C}_{3}, \mathrm{C}_{4}, \mathrm{C}_{6} \mathrm{H}_{4}$ ). The large impact on the mole fraction of $\mathrm{NH}_{3}$ comes here from the dissociative recombination of $\mathrm{CH}_{3} \mathrm{CNH}^{+}$, for which the $\mathrm{BR}$ set is incomplete $[11,28]$. For $\mathrm{C}_{6} \mathrm{H}_{6}$, many processes are involved with small contributions, and no dominant source has been identified yet.

\section{Conclusion}

This study evaluates directly the importance of implementing a full representation of dissociative recombination branching ratios in models of $\mathrm{N}_{2} / \mathrm{CH}_{4}$ photochemical plasmas. A significant effect of this representation on the mole fractions of products is observed in two typical scenarii. The effect is enhanced in the experimental setup conditions at higher pressure. In this case, it has been shown that the "H-loss" model misses major pathways of $\mathrm{C}_{2} \mathrm{H}_{2}$ production, with large impact on molecular growth promoted by this species.

The data request of Yelle et al. [27] for the branching ratios of $\mathrm{CH}_{2} \mathrm{NH}_{2}^{+}$to predict correctly $\mathrm{NH}_{3}$ abundance is confirmed, and we have shown that it has a more global impact on many N-bearing species. More generally, as the dissociation of ions into smaller neutrals, notably $\mathrm{C}_{2} \mathrm{H}_{x}$ unsaturated species has a strong impact on molecular growth, the missing breakup patterns of "heavy" $\mathrm{C}_{x-y} \mathrm{~N}_{y} \mathrm{H}_{z}^{+}(x \geq 5)$ ions are urgently needed, even with unresolved $\mathrm{H}$ atoms.

\section{References}

[1] J.B.A. Mitchell, G. Angelova, C. Rebrion-Rowe, O. Novotny, J.L.L. Garrec, H. Bluhme, K. Seiersen, A. Svendsen, L.H. Andersen, Journal of Physics: Conference Series 4, 198 (2005)

[2] M. Larsson, A. Orel, Dissociative Recombination of Molecular Ions (Cambridge University Press, 2008)

[3] C. Keller, T. Cravens, L. Gan, Journal of Geophysical Research 97, 12117 (1992)

[4] N. Keller, V. Anicich, T. Cravens, Planetary and Space Science 46, 1157 (1998)

[5] M. Banaszkiewicz, L. Lara, R. Rodrigo, J. Lopez-Moreno, G. Molina-Cuberos, Icarus 147, 386 (2000)

[6] E.H. Wilson, S.K. Atreya, Journal of Geophysical Research 109, E06002 (2004)

[7] V. De La Haye, J. Waite, T. Cravens, I. Robertson, S. Lebonnois, Icarus 197, 110 (2008)

[8] V. Krasnopolsky, Icarus 201, 226 (2009)

[9] A.I. Florescu-Mitchell, J.B.A. Mitchell, Physics Reports 430, 277 (2006)

[10] E. Vigren, J. Semaniak, M. Hamberg, V. Zhaunerchyk, M. Kaminska, R.D. Thomas, M. af Ugglas, M. Larsson, W.D. Geppert, Planetary and Space Science 60, 102 (2012)

[11] S. Plessis, N. Carrasco, P. Pernot, J. Chem. Phys. 133, 134110 (2010)

[12] P. Pernot, S. Plessis, N. Carrasco, Journal of Physics: Conference Series 300, 012027 (2011)

[13] S. Plessis, N. Carrasco, M. Dobrijevic, P. Pernot, Icarus 219, 254 (2012)

[14] Z. Peng, Experimental and Theoretical Simulations of Titan's VUV Photochemistry, Ph.D. Thesis, Univ. Paris-Sud, France (2013)

[15] Z. Peng, T. Gautier, N. Carrasco, P. Pernot, A. Giuliani, A. Mahjoub, J.-J. Correia, A. Buch, Y. Bénilan, C. Szopa, and G. Cernogora, The Journal of Geophysical Research: Planets 118, 778 (2013)

[16] N. Carrasco, J. Westlake, P. Pernot, H. Waite, Jr, Astrophysics and Space Science Proceedings 35, 145 (2013)

[17] H. Imanaka, M.A. Smith, Geophysical Research Letters 34, L02204 (2007)

[18] H. Imanaka, M.A. Smith, Proceedings of the National Academy of Sciences 107, 12423 (2010)

[19] Z. Peng, T. Gautier, N. Carrasco, P. Pernot, A. Giuliani, A. Mahjoub, J.J. Correia, A. Buch, Y. Bénilan, C. Szopa et al., Journal of Geophysical Research: Planets 118, 778 (2013) 
[20] A. Giuliani, F. Jamme, V. Rouam, F. Wien, J.L. Giorgetta, B. Lagarde, O. Chubar, S. Bac, I. Yao, S. Rey et al., Journal of Synchrotron Radiation 16, 835 (2009)

[21] E. Hébrard, M. Dobrijevic, Y. Bénilan, F. Raulin, Journal of Photochemistry and Photobiology A: Chemistry 7, 211 (2006)

[22] E. Hébrard, M. Dobrijevic, P. Pernot, N. Carrasco, A. Bergeat, K.M. Hickson, A. Canosa, S.D. Le Picard, I.R. Sims, The Journal of Physical Chemistry A 113, 11227 (2009)

[23] B. Gans, Z. Peng, N. Carrasco, D. Gauyacq, S. Lebonnois, P. Pernot, Icarus 223, 330 (2013)

[24] N. Carrasco, C. Alcaraz, O. Dutuit, S. Plessis, R. Thissen, V. Vuitton, R. Yelle, P. Pernot, Planetary and Space Science 55, 1644 (2008)

[25] M. Larsson, A. Ehlerding, W.D. Geppert, F. Hellberg, S. Kalhori, R.D. Thomas, N. Djuric, F. Osterdahl, G. Angelova, J. Semaniak et al., The Journal of Chemical Physics 122, 156101 ( 3) (2005)

[26] R. Thissen, V. Vuitton, P. Lavvas, J. Lemaire, C. Dehon, O. Dutuit, M.A. Smith, S. Turchini, D. Catone, R.V. Yelle et al., J. Phys. Chem. A 113, 11211 (2009)

[27] R.V. Yelle, V. Vuitton, P. Lavvas, S.J. Klippenstein, M.A. Smith, S.M. Hörst, J. Cui, Faraday Discuss. 147, 31 (2010)

[28] E. Vigren, M. Kaminska, M. Hamberg, V. Zhaunerchyk, R.D. Thomas, M. Danielsson, J. Semaniak, P.U. Andersson, M. Larsson, W.D. Geppert, Physical Chemistry Chemical Physics 10, 4014 (2008) 